\title{
ВОЗМОЖНОСТИ НЕИНВАЗИВНОЙ ДИАГНОСТИКИ ИЗМЕНЕНИЙ ВЕНЕЧНЫХ АРТЕРИЙ У БОЛЬНЫХ САХАРНЫМ ДИАБЕТОМ
}

\author{
М. Д. Султанова \\ Азербайджанский медицинский университет, г. Баку, Республика Азербайджан

\section{POSSIBILITIES OF NONINVASIVE DIAGNOSIS OF THE CORONARY ARTERIES CHANGES IN PATIENTS, SUFFERING DIABETES MELLITUS}

\author{
M. D. Sultanova
}

Azerbaijan Medical University, Baku, Republic of Azerbaijan

\begin{abstract}
Реферат
Цель. Оценить степень кальцификации венечных артерий (ВА) у больных сахарным диабетом (СД) по данным цифровой рентгенографии (ЦР) и мультиспиральной компьютерной томографии (МСКТ).

Материалы и методы. Ретроспективно проанализированы результаты обследования 29 больных СД II типа. При ЦР органов грудной полости кальцификация выявлена у 11 (37,9\%) больных - в одной, у $10(34,5 \%)$ - в двух, у $6(20,7 \%)$ - в трех и более ВА. При МСКТ кальцификация выявлена у 4 (13,8\%) больных - в одной, у $10(34,5 \%)$ - в двух, у 15 (51,7\%) - в трех и более ВА. Результаты. Частота кальцификации ВА зависела от возраста пациентов и продолжительности заболевания. Больным СД при предположении о наличии ишемической болезни сердца (ИБС) показано проведение ЦР и МСКТ, которые позволяют получить ценную информацию, подтвердить кальцификацию ВА для обоснования тактики лечения.

Ключевые слова: сахарный диабет; кальцификация венечных артерий; диагностика; цифровая рентгенография; мультиспиральная компьютерная томография.

\section{Abstract}

Objective. To estimate a degree of the coronary arteries calcification in patients, suffering diabetes mellitus, in accordance to data of digital roentgenorgaphy and multispiral computer tomography.

Materials and methods. Results of examination of 29 patients, suffering diabetes mellitus type II, were analyzed retrospectively. In thoracic digital roentgenography calcification was revealed in $11(37.9 \%)$ patients - in one, in $10(34.5 \%)$ - in two, and in $6(20.7 \%)$ - in three and more coronary arteries.

In multispiral computer tomography calcification was revealed in $4(13.8 \%)$ patients -in one, in $10(34.5 \%)$ - in two, and in $15(51.7 \%)$ - in three and more coronary arteries.

Results. The coronary arteries calcification rate have depended upon age of the patients and duration of the disease. Conduction of digital roentgenorgaphy and multispiral computer tomography, which permit to obtain valid information and approve presence of calcification of coronary arteries for substantiation of the treatment tactics, is indicated in patients with diabetes mellitus with suspicion for the ischemic heart disease presence.

Keywords: diabetes mellitus; calcification of coronary arteries; diagnosis; digital roentgenography; multispiral computer tomography.
\end{abstract}

Заболеваемость СД ІІ типа увеличивается с каждым годом, будучи одной из актуальных проблем современного здравоохранения. Наиболее частой причиной смерти взрослых пациентов при СД является ИБС [1], примерно 60 - 75\% - умирают вследствие атеросклероза ВА, 10 25\% - атеросклероза церебральных и периферических артерий $[2,3]$.

Установлена связь между повышением уровня глюкозы в крови и увеличением заболеваемости и смертности от сердечно-сосудистых заболеваний $[4,5]$.

У больных СД ІІ типа частота ИБС в 2 - 4 раза, риск возникновения острого инфаркта миокарда - в 6 10 раз, мозгового инсульта - в $4-7$ раз выше, чем у пациентов без СД [6].
Роль СД в формировании атеросклероза, который при этом заболевании начинается раныше и протекает тяжелее, не вызывает сомнения. Атеросклероз у таких больных возникает на 20 лет раньше, стремительно прогрессирует и имеет характерные клинические проявления, в отличие от пациентов без СД. У пациентов при СД преимущественно возникает безболевая форма ИБС, в связи с чем изменения ВА выявляют несвоевременно, что обусловливает высокий риск осложнений и позднюю терапию ИБС. У 36\% больных, госпитализированных в неотложном порядке по поводу острого коронарного синдрома, у которых ранее не было нарушений углеводного обмена, выявлены при- знаки предиабета (нарушение гликемии натощак, толерантности к глюкозе), у 22\% - СД II типа [7].

Результаты коронарной ангиографии у больных СД и без него противоречивы. Некоторые авторы отмечают одинаково частое повреждение сосудов у пациентов обеих групп, другие - подчеркивают более тяжелое поражение сосудов у больных СД [8 - 11].

В настоящее время оценку состояния ВА у пациентов при СД с применением неинвазивных методов исследования считают более актуальной. Прогресс в развитии радиологических методов исследования изменил роль рентгенографии в диагностическом процессе. В течение длительного времени первичным 
и основным этапом в диагностическом поиске считали рентгенологические методы, однако с появлением КТ, МРТ, ПЭТ КТ и других высокотехнологических методов исследования ситуация изменилась.

Несмотря на широкое использование приведенных методов исследования, ЦР органов грудной полости играет важную роль в определении групп риска ИБС у пациентов при СД. Клинически доказано, что диагностика заболеваний ВА с использованием этого метода несколько ограничена, поэтому метод может быть использован для визуализации кальцификации ВА только высокой плотности. С этой точки зрения целесообразно использование рентгенографии для выявления групп риска пациентов с сердечнососудистыми заболеваниями. Подтверждением этому являются многочисленные научные исследования, посвященные широкому применению рентгенографии в диагностике кальцификации ВА $[12,13]$.

Возможность получения изображения в различных режимах дает большие преимущества в дифференциации кальцификации BA и мягких тканей грудной полости с высокой точностью.

В последнее время МСКТ ВА стала информативным методом выявления ИБС, особенно у пациентов при средней степени риска ее возникновения или в ситуациях, когда тесты на наличие ишемии сомнительны. В исследованиях, посвященных диагностической точности МСКТ в выявлении ИБС по сравнению с таковой инвазивной коронарной ангиографии, отмечены высокая чувствительность и прогностическая точность, близкая к 100\%. Возможность выявлять не только стеноз ВА, но и атеросклеротические бляшки неин- вазивным путем делает МСКТ особенно ценным методом в стратификации риска [14, 15].

Целью исследования было изучение состояния ВА у больных СД с помОщью ЦР и МСКТ.

\section{МАТЕРИАЛЫ И МЕТОДЫ ИССЛЕДОВАНИЯ}

Обобщены результаты ЦР и МСКТ грудной полости у 29 больных СД II типа, в том числе 14 (48,3\%) мужчин и 15 (51,7\%) женщин. В возрасте до 50 лет было 3 (10,3\%) больных, от 51 до 60 лет - 7 (24,1\%), от 61 до 70 лет 19 (65,5\%). Продолжительность СД II типа у 5 (17,2\%) пациентов - до 3 лет, у $14(48,2 \%)-4$ - 9 лет, у $10(34,4 \%)-$ более 9 лет.

Диагноз СД устанавливали при уровне глюкозы натощак 126 мг/дл и более. Лечение СД включало диету, таблетированные сахароснижающие препараты. Факторами риска считали: артериальную гипертензию (артериальное давление выше 18,7/12,0 кПа, или 140/90 мм рт. ст.) либо сведения в анамнезе об использовании постоянно антигипертензивных препаратов; гиперхолестеринемию (уровень общего холестерина 200 мг/дл или постоянное применение липидоснижающих препаратов); ожирение (индекс массы тела более 30 кг/м²); семейный анамнез по ИБС, курение (в прошлом или в настоящее время).

У всех больных проведена ЦР грудной полости в 2 стандартных проекциях с использованием цифрового рентгеновского аппарата Flexavision Shimadzu (Япония). Рентгенограммы обработаны с помощью компьютерной системы Fujifilm FCR Capsula XL II для лучшей оценки peзультатов.

МСКТ проведена на КТ сканере Toshiba (128 slice). В начале получа- ли нативные изображения, синхронизированные с ЭКГ. Эти сканы использовали для определения кальциевого индекса Агатстона. Затем, после внутривенной инъекции 80 100 мл неионного йодсодержащего контрастного вещества, проводили МСКТ. При частоте сокращений сердца более 65 в 1 мин дополнительно назначали внутривенно бета-блокаторы (атенолол 5 - 10 мг).

С использованием специализированного программного обеспечения у каждого пациента определяли общий кальциевый индекс Агатстона, кальцинат в ВА определяли как участок площадью более 1 мм² $^{2}$ плотностью более 130 HU. Все 16 сегментов, классифицированных Американской Ассоциацией Сердца, включены в анализ.

Для статистической оценки результатов проведен корреляционный анализ методом $\chi^{2}$ Пирсона.

\section{РЕЗУЛЬТАТЫ \\ И ИХ ОБСУЖДЕНИЕ}

При оценке рентгенограмм у всех больных визуализирована кальцификация ВА различного характера и локализации.

По данным ЦР у 11 (37,9\% ะ 9,0\%) больных кальцификация выявлена в одной ВА, у 10 (34,5\% $\pm 8,8 \%)$ - в двух, у $6(20,7 \% \pm 7,5 \%)$ - в трех и более ВА. У 17 (58,6\% $\pm 9,1 \%)$ больных визуализирована кальцификация дуги аорты, у 1 (3,4\% \pm 7,0\%) больного - митрального клапана, у 1 (3,4\% \pm 7,0\%) - перикарда.

У 19 (65,5\% 土 8,8\%) больных визуализирована кальцификация в передней межжелудочковой ветви левой BA (LDA), у $17(58,6 \% \pm 9,1 \%)-$ в огибающей ветви левой BA (LCx), у 8 $(27,6 \% \pm 8,3 \%)-$ в правой BA (RCA), у $2(6,9 \% \pm 4,7 \%)-$ в основном стволе левой BA (LMA).

Частота выявления кальцификации ВА у пациентов разного возраста

\begin{tabular}{|c|c|c|c|c|c|c|c|c|}
\hline \multirow{4}{*}{ Количество ВА } & \multicolumn{8}{|c|}{ Число больных в возрасте, лет по данным } \\
\hline & \multicolumn{4}{|c|}{ ЦР } & \multicolumn{4}{|c|}{ MCKT } \\
\hline & \multicolumn{2}{|c|}{ до $60(n=10)$} & \multicolumn{2}{|c|}{ старше $60(n=19)$} & \multicolumn{2}{|c|}{ до $60(n=10)$} & \multicolumn{2}{|c|}{ старше $60(n=19)$} \\
\hline & абс. & $\%$ & абс. & $\%$ & абс. & $\%$ & абс. & $\%$ \\
\hline 1 & 8 & 80,0 & 3 & 15,8 & 4 & 40,0 & - & - \\
\hline 2 & - & & 10 & 52,6 & 4 & 40,0 & 6 & 31,6 \\
\hline 3 и более & - & & 6 & 31,6 & 2 & 20,0 & 13 & 68,4 \\
\hline$\chi^{2} ; p$ & \multicolumn{4}{|c|}{$r=0,555 ; \chi^{2}=19,3 ; p<0,01$} & \multicolumn{4}{|c|}{$r=0,346 ; \chi^{2}=10,7 ; p<0,01$} \\
\hline
\end{tabular}


По данным МСКТ у 4 (13,8\% \pm 6,4\%) больных кальцификация обнаружена в одной, у 10 (34,5\% 土 8,8\%) - в двух, у 15 (51,7\% \pm 9,3\%) - в трех и более ВА.

У 29 (100\%) больных кальцификация визуализирована в LDA, у 24 $(82,8 \% \pm 7,0 \%)-$ в LCx, у $15(51,7 \% \pm$ $9,3 \%)$ - в RCA, у 5 (17,2\% $\pm 7,0 \%)-$ в LMA; у 25 (86,2\% $\pm 6,4 \%)$ - визуализирована кальцификация дуги аорты; митрального клапана, перикарда по одному наблюдению.

При МСКТ кальциевый индекс Агатстона составил в среднем 375,5 HU.

При сравнительной оценке результатов ЦР и МСКТ отмечено, что не определяемые по данным ЦР мягкие смешанные формы атеросклеротических бляшек выявляли по всей сети ВА. Поскольку их количество составляло в LDA - 10, в LCX - 7,

\section{REFERENCES}

1. Malmberg K, Rydén L, Wedel H, Birkeland K, Bootsma A,Dickstein $\mathrm{K}$, et al. Intense metabolic control by means of insulin in patients with diabetes mellitus and acute myocardial infarction (DIGAMI 2): effects on mortality and morbidity. Eur Heart J. 2005 Apr;26(7):65061. Epub 2005 Feb 23.

2. Karpov YUA. Mozhno li uspeshno lechit bolnykh ishemicheskoy boleznyu serdtsa v sochetanii s sakharnym diabetom. Sakharnyy diabet. 2002;(4):24-8. [In Russian].

3. Mukamal KJ, Nesto RW, Cohen MC, et al. Comparability of risk with prior myocardial infarction. Diabetes Care. 2001;24(8):1422-7.

4. Gerstein HC, Miller ME, Byington RP, et al. Effects of intensive glucose lowering in type 2 diabetes. New Engl J Med. 2008 Jun 12;358(24):2545-59. doi: 10.1056/NEJMoa0802743. Epub 2008 Jun 6.

5. Selvin E, Marinopoulos S, Berkenblit G, et al. Meta-analysis: glycosylated hemoglobin and cardiovascular disease in diabetes mellitus. Ann Intern Med. 2004;141:421-31. PMID: 15381515

6. Biryukova EV. Samokontrol glikemii - deystvennaya mera profilaktiki serdechno-sosudistykh zabolevaniy u bolnykh sakharnym diabetom. Meditsinskiy sovet.2014;12:112-15. [In Russian].

7. Bartnik M, Rydén L, Ferrari R, Malmberg K, Pyörälä K, Simoons M, et al. The prevalence of abnormal glucose regulation in patients with coronary artery disease across Europe. The Euro Heart Survey on diabetes and the heart. Eur Heart J. 2004;25:1880-90. PMID: 15522466

8. Azizov VA, Sultanova MD. Osobennosti porazheniya koronarnykh sosudov u bolnykh s ishemicheskoy boleznyu serdtsa na fone sakhar- nogo diabeta. Ukrainskyi zhurnal klinichnoi ta laboratornoi medytsyny. 2013; 8(2):109-12. [In Russian].

9. Alhamadani DA, Husain FY, Abbo MA. Coronary angiographic findings among diabetic and non-diabetic patients. Annals of the College of Medicine. 2009;35(1):65-72.

10.Salim M. AL-Rubae MBChB. Evaluating coronary artery disease in type 2 diabetes mellitus by angiographic study. J Fac Med Baghdad. 2011;53(1):15-9.

11. Scognamiglio R, Negut C, Ramondo A, et al. Detection of coronary artery disease in asymptomatic patients with type 2 diabetes mellitus. J Am Coll Cardiol. 2006;47:65-70.

12. Baris A, Mustafa S, Cetin Y, Erhan A. The relationship between aortic knob width and various demographic, clinical, and laboratory parameters in stable hemodialysis patients. Saudi J Kidney. Dis Transpl. 2014;25;1178-85.

13. Elif T, Fehmi K. Clinical implications of chest $x$-ray parameters in evaluating patients with cardiac dyspnea. The Eurasian Journal of Medicine. 2008;40:133-6.

14. Ternovoy SK, Sinitsyn VE, Gagarina NV. Neinvazivnaya diagnostika ateroskleroza i kaltsinoza koronarnykh arteriy. Moskva: Atmosfera; 2003.140 c.

15. Sun Z, Aziz YF, Ng KH. Coronary CT angiography: how should physicians use it wisely and when do physicians request it appropriately? Eur J Radiol. 2012 Apr;81(4):e684-7. doi: 10.1016/j. ejrad.2011.06.040. Epub 2011 Jul 2.

В журналі Клінічна хірургія № 4 за 2016 рік на сторінках 4 та 67 замість «Dynamics of bacteriological and planimetric indices of the wound under the action of the silver nanoparticles, stabilized by mexidol and polyvinylpyrrolidone.

Lyakhovskyi V. I., Lobahn G. A., Gancho O. V., Vazhnycha O. M., Kolomiyets S. V., Jaber V. Kh. O.» слід читати

«Dynamics of bacteriological and planimetric indices of the wound under the action of the silver nanoparticles, stabilized by mexidol and polyvinylpyrrolidone.

Lyakhovskyi V. I., Loban G. A., Gancho O. V., Vazhnycha O. M., Kolomiyets S. V., Jaber V. Kh. O.» 